\title{
Benefícios, prejuízos e considerações relevantes na utilização de sistemas de recifes artificiais e estruturas correlatas *
}

\author{
Benefits, damages and relevant considerations in the use of \\ artificial reef and correlated structure systems
}

\author{
G. Castanhari ${ }^{\circledR, 1}$, A. R. G. Tomás ${ }^{1}$, C. I. Elliff ${ }^{1}$
}

\section{RESUMO}

Recifes artificiais e estruturas correlatas têm sido usadas ao redor do mundo como ferramentas de manejo para melhorar a pesca e turismo e mitigar áreas impactadas, além de outras funçôes. No entanto, projetos envolvendo recifes artificiais requerem uma série de estudos e um planejamento adequado para que sua implantação não cause danos ao meio ambiente, como alteraçôes severas nas comunidades biológicas marinhas locais e adjacentes assim como nos padróes oceanográficos locais. Existe ainda, no caso de estruturas mal planejadas, o risco de perdas de partes desta estrutura devido às correntes, subsidência ou movimentos não desejados, danos a rotas de navegação e conflitos entre diferentes grupos de usuários. Assim, qualquer tentativa de implantar projetos usando recifes artificiais e estruturas correlatas deve ser seguida de uma reflexão sobre sua relevância, planejamento e monitoramento eficiente e uma avaliaçáo aprofundada quanto seu impacto em potencial.

Palavras chave: gerenciamento costeiro; pesca; avaliação de impactos

\section{ABSTRACT}

Artificial reefs and correlated structures have been largely used all over the world as management tools to enhance fisheries and tourism and mitigate damaged areas, among others functions. Nevertheless, projects involving artificial reefs require a series of studies and an adequate planning so its implementation will not cause any damage to the environment, such as severe alterations on the local and surrounding marine biological communities as well as alterations on the local oceanographic patterns. There is, still, on not well planned ventures, the risk of lost of parts of the structures due to scouring, subsidence or undesirable movement, damage to navigation routes, and conflicts among different groups of users. Thus, any attempt to implement projects using artificial reefs and correlated structures must be followed by reflection about its relevance, efficient planning and monitoring and a deep evaluation of its potential impact.

Keywords: coastal management; fisheries; impact assessment

@ - Corresponding author

1 - Instituto de Pesca, Av Bartolomeu de Gusmão, 192, Santos - SP 11030-906, Brasil.E-MAILS: grazi.castanhari@ig.com.br,argtomas@pesca.sp.gov.br, carlaelliff@gmail.com 


\section{INTRODUÇÃO}

Definem-se "recifes artificiais" como estruturas inseridas no meio aquático acidental ou propositalmente, a fim de fornecer substrato consolidado que permita a fixação de espécies sésseis, criando a oportunidade de surgimento de toda uma comunidade biológica a partir da incrustação de produtores primários. Este incremento de biomassa ocorre pelo aumento da disponibilidade de alimento, promoção de habitat para recrutamento e pontos de encontro para a reproduçáo (Bohnsack, 1989; White et. al., 1990). De acordo com o Conselho Nacional do Meio Ambiente (CONAMA) do Ministério do Meio Ambiente (MMA) do Brasil, recifes artificiais são definidos como qualquer estrutura construída ou preparada para instalação em ambiente subaquático que simulam as propriedades de recifes naturais, tendo como principais finalidades: conservação, manejo e pesquisa; e exploração e o aproveitamento sustentável dos recursos do mar. Pitcher e Seaman (2001) discutem sobre usar o termo "artificial” e sua possível conotação negativa, preferindo então chamar essas estruturas de "human-made reefs" - recifes feitos pelo homem.

Recifes artificiais e estruturas correlatas (RAECs) podem ser construídos de uma enorme variedade de materiais, que vão desde materiais naturais, como rochas e bambus, a um tipo de concreto desenvolvido especialmente para a construção de recifes artificiais marinhos. A configuração das estruturas e o material utilizado nos módulos influenciam a eficácia dos sistemas, em aspectos como resistência às condições ambientais, composição de espécies, durabilidade e viabilidade da implantação do projeto.

A utilização destas estruturas para o incremento de atividades pesqueiras teve início em épocas remotas, quando pescadores notaram uma maior concentração de peixes ao redor de estruturas flutuantes (Seaman e Sprague, 1991a). Whitmarsh et al. (2008) afirmam que o uso de estruturas físicas submersas para aumentar a abundância de vida marinha provavelmente deu-se ao acaso.

A percepção deste fato aliada ao interesse em aprimorar a utilização de tais estruturas fez com que recifes artificiais e estruturas correlatas se constituíssem em tema de inúmeros estudos no mundo inteiro. Isso os tornou ferramentas interessantes no manejo de áreas pesqueiras, por criarem oportunidades de pesca e diminuírem o tempo de viagem pela procura dos recursos aumentando a eficiência do petrecho de pesca (Polovina, 1991; Svane e Petersen, 2002). Esse interesse crescente no desenvolvimento de habitats artificiais para atividades pesqueiras levou os gerenciadores, que inicialmente focavam encontrar metodologias de despejo e tipos de materiais que criariam recifes eficazes, a se preocupar sobre seu desempenho biológico e econômico (Moffit et al., 1989; Steimle e Meier, 1997).

A popularizaçáo de seu uso nas atividades pesqueiras abriu caminhos para outros tipos de utilização. Atualmente, RAECs podem funcionar, por exemplo, como ferramentas de mitigação de áreas degradadas e como atrativos para áreas utilizadas por mergulhadores recreativos (Polovina, 1991; Seaman e Sprague, 1991b; Santos et al., 2010). $\mathrm{O}$ uso de RAECs também pode auxiliar na contenção da pesca de arrasto, em locais aonde esta prática é proibida, pois esta inviabiliza o uso da rede no local, causando danos à propriedade do infrator (Claudet e Pelletier, 2004; Whitmarsh et al., 2008). Também é possível proporcionar melhores condiçóes para a prática do surfe, através de estruturas que alteram as características da ondulação de uma região, além de usá-las como mecanismos de defesa da costa (Voorde et al., 2009; Voorde et al., 2008). Outros autores tem adotado denominação assemelhada para definir estruturas que tenham como objetivos tanto a atenuar impactos como promover uso sustentável do ambiente, os recifes artificiais multifuncionais (Carmo et al., 2010)

A ecologia de comunidades recifais é um dos temas mais abordados na pesquisa científica relativa ao uso de RAECs (como em Athie, 1999; Conceição, 2003), porém o manejo pesqueiro é apontado como o principal fator na decisão da implantação de um (Baine, 2001; Wilson et al., 2002; Miller, 2002; Svane e Petersen, 2002; Quintero, 2009). No entanto, Bortone (2006) afirma que não há RAECs que sejam formalmente usados para incrementar a atividade pesqueira, denotando uma necessidade de efetivamente incorporar os RAECs no manejo pesqueiro.

A demanda por estes projetos tem crescido no mundo inteiro, sendo foco de diversas pesquisas científicas (Carmo et al., 2010). Baine (2001) observou a distribuição global dos trabalhos revisados por ele e constatou que a maioria se concentrou na América do Norte (38\%). O Japáo representou apenas $12 \%$ dos trabalhos revisados, apesar de ser reconhecido como o país líder em pesquisa sobre recifes artificiais. De acordo com Svane e Petersen (2002), a maioria dos estudos sobre RAECs são realizados em regióes subtropicais e tropicais, criando um acúmulo de conhecimento ecológico apenas nessas áreas.

Apesar do grande interesse pelo tema, relativamente pouco se é conhecido sobre a biologia e ecologia destas estruturas e nem sempre sua implantação é a solução para o problema em mãos (Baine, 2001). RAECs implantados sem os cuidados necessários podem causar impactos negativos, como alteração na linha da costa, perda de estruturas pela inadequação do substrato, conflitos entre grupos de usuários distintos e até mesmo depleção de estoques pela pesca desordenada (Polovina, 1991; ASMFC, 1998; Baine, 2001; Santos et al., 2010; Carmo et al., 2010). Collins et al. (2002) e Quintero (2009) discutem como a escolha do material de construção dos RAECs pode vir a ter um impacto negativo através da exposição da biota a substâncias químicas e causar poluiçáo do meio.

Bohnsack e Sutherland (1985) revisaram a literatura a respeito, constatando que a maioria dos trabalhos publicados sobre o assunto são descrições qualitativas, detalhando modificaçôes na sucessão ecológica e as espécies observadas. Poucos estudos usam métodos experimentais quantitativos e em muitos há falta de controles científicos válidos. Steimle e Meier (1997) e Svane e Petersen (2002) afirmam que, apesar do recente aumento no número de estudos, estes tendem a ter uma visão restrita. Polovina (1991) e, mais recentemente, Sutton e Bushnella (2007) e Whitmarsh et al. (2008) ressaltam, ainda, a falta de estudos socioeconômicos e de gerenciamento mais amplos e destacam a importância de medidas que regulamentem os petrechos e o esforço pesqueiro para evitar sobrepesca. Segundo estes autores, 
para haver progresso no entendimento das aplicaçóes de projetos utilizando RAECs, estes devem ser instalados como parte de um plano de manejo abrangente, condizente com as limitações de cada habitat artificial em particular. Baine (2001) apontou que a maior parte dos problemas relatados com o uso de RAECs em estudos diz respeito ao seu planejamento e questóes de manejo.

A escolha do local para implantar RAECs também ocorre em função de diversos fatores. A participação das comunidades locais e dos grupos que utilizarão a área é essencial (Santos et al., 2010; Quintero, 2009). Barber et al. (2009) criaram um modelo de mapeamento de exclusão sistemático, onde considerou-se também os parâmetros físicos e biológicos da área, como a profundidade, declividade, composiçâo do substrato, acessibilidade, correntes, ação de ondas, habitats estabelecidos próximos, fornecimento natural de larvas, qualidade da água e conflito entre usuários.

No Brasil, a demanda por projetos utilizando RAECs também é crescente (Santos et al., 2010), porém os projetos nem sempre consideram todos os fatores mencionados. Santos et al. (2010) relataram os conflitos de interesse da instalaçáo de RAECs no litoral do Estado de Pernambuco. A falta de dados para que aspectos importantes, como o comportamento das estruturas artificiais e seus impactos ambientais e socioeconômicos, inviabiliza análises mais completas que possam contribuir para a melhor aplicação destas estruturas e para o aprimoramento da implantaçấo e manejo das mesmas, potencializando impactos negativos de sua implantação.

\section{DISCUSSÃO}

\subsection{Funcionamento das Estruturas Artificiais}

Quaisquer habitats artificiais incrementam os sistemas marinhos. Este incremento acontece pelo adicionamento de área de superfície e espaços criados pelas estruturas na coluna d'água que permitem que organismos marinhos se fixem e encontrem abrigo (White et al., 1990). RAECs podem agir na composição da ictiofauna por fornecer alimento adicional, aumentar a eficiência da alimentação, fornecer abrigo contra predação, fornecer habitat para recrutamento e criar espaços vagos em ambientes naturais (Bohnsack, 1989).

As estruturas artificiais fornecem também abrigo e áreas de descanso para os peixes em relação às condições oceanográficas da região. Quando a velocidade da corrente aumenta, os peixes tendem a se abrigar na ondulação lee (lee wave) formada pelas estruturas (Nakamura, 1985; Baynes e Szmant, 1989). Essa ondulação pode sofrer interferência do aumento na velocidade da corrente, dando lugar a uma área protegida (plain wake), formando uma "sombra" da corrente e de tempestades que causam uma mistura vertical na coluna d'água. Nestas condições, os peixes migram para as camadas medianas e inferiores da coluna d'água (Nakamura, 1985). Sons emitidos pelos organismos no recife e flutuaçóes de pressão devido à turbulência associada à corrente impingindo nas estruturas podem estimular os peixes a se dirigirem às estruturas (Nakamura, 1985; Baynes e Szmant, 1989; Grove et al., 1991).

Os padrôes de comportamento dos peixes estáo relacionados a hábitos instintivos de orientar os movimentos em resposta a vários estímulos. Este estímulo constitui a ligação fundamental entre os peixes e os recifes (Nakamura, 1985). Este autor classifica os peixes recifais em três categorias:

- tipo A: estabelecem contato físico com o recife, ocupando locas, fendas e aberturas extensas; se alimentam predominantemente de organismos bentônicos.

- tipo B: são ligados às estruturas pelo som e visão; nadam ao redor dos recifes enquanto permanecem próximos ao fundo.

- tipo C: nadam ao redor dos recifes enquanto permanecem nas partes superiores e intermediárias da água.

Athiê (1999) elaborou uma nova classificação de comportamento das espécies, baseada nestas categorias, considerando o tempo de permanência dos peixes nas estruturas:

- Colonizadoras: após o primeiro registro de sua ocorrência, ocorre um aumento gradual de freqüência e abundância até que atinja o equilíbrio de sua população; nesta categoria estáo inclusas as espécies que uma vez registradas nas estruturas são vistas regularmente, de forma constante; são as espécies que utilizam as estruturas para residência, alimentação e reprodução, povoando o recife;

- Exploradoras: apresentam freqüências de ocorrência moderada a alta, podendo ou não apresentar grande abundância, porém sem haver aumento gradual nestas freqüências; apresentam também registros de ocorrência mais esparsos, não sendo residentes dos recifes, utilizando o recife para alimentaçáo, proteção e possível reprodução sem, no entanto, fixar moradia ou criar vínculo de dependência com as estruturas;

- Ocasionais: apresentam freqüências de ocorrência baixas, podendo, ou não, serem registradas em grande abundância, não apresentando aumentos graduais nestas freqüências; apresentam registros muito descontínuos e eventuais, por ocorrerem raramente nos recifes; espécies que estâo no recife de passagem, não apresentando nenhuma relação cíclica ou duradoura de ocorrência junto às estruturas.

Hixon e Beets (1989) encontraram uma relação estatística negativa entre o número de residentes piscívoros e o número de peixes pequenos nos recifes, sugerindo que a quantidade de piscívoros determina o número de peixes pequenos nas estruturas. Ainda de acordo com esses autores, em grandes recifes, a proporção de abundância de abrigo e de alimento pode diminuir para alguns peixes de passagem, especialmente planctófagos, o que tenderia a aumentar a importância relativa de fatores tróficos na determinação da abundância e distribuição local de peixes. De maneira geral, existe uma correlação positiva entre complexidade estrutural e abundância e diversidade de espécies de peixes (Sherman et al., 2002).

Recifes artificiais são interessantes para testar hipóteses a respeito de estruturas de abrigo, pois permitem a separação dos efeitos da alimentação e do abrigo, o controle da estrutura 
e do histórico do recife e, mais importante, controlar os experimentos com réplicas verdadeiras e tratamento aleatório (Hixon e Beets, 1989). Matthews (1985) e D'Anna et al. (1994) encontraram uma composição de ictiofauna similar entre recifes artificiais e recifes naturais, porém Zalmon et al. (2002) encontrou uma riqueza maior no recife artificial analisado.

\subsection{Aspectos Relevantes na Elaboraçáo de Projetos Utilizando RAECs}

\section{A) Configuração das estruturas}

Os modelos de RAECs pioneiros, desenvolvidos há séculos por pescadores artesanais eram constituídos de materiais naturais, como bambus, folhas e toras de madeira. $\mathrm{Na}$ regiáo Nordeste do Brasil, pescadores usam há geraçóes as chamadas marambaias, que são aglomerados de diversos materiais (p.ex.: madeira de mangue) jogados no fundo marinho (Conceição, 2003; Quintero, 2009).

Com a evolução da pesquisa sobre o desenvolvimento de habitats artificiais, novos materiais foram testados e, atualmente, uma grande variedade destes é utilizada, como pneus, concreto, carcaças, tubos de PVC, entre outros. Lukens (1997) faz uma descrição detalhada dos principais materiais comumente empregados na confecção de habitats artificiais, relatando os benefícios e prejuízos da utilização de cada um deles:

\section{- Concreto}

- Benefícios: Alta compatibilidade com o meio ambiente marinho, durabilidade, estabilidade, pronta disponibilidade, facilidade de se obter a forma desejada, boa superfície para fixação e desenvolvimento de organismos incrustantes.

- Prejuízos: Material pesado, o que pode levar a afundamento no substrato e dificulta o transporte e fundeio, dependendo de equipamentos pesados para a implantação do módulo. Peças pequenas podem ser difíceis de serem empilhadas durante a implantaçấo.

- Pneus

- Benefícios: Grande disponibilidade, baixo preço de aquisição, durabilidade, boa capacidade de atrair fauna.

- Prejuízos: Dificuldade de instalação de organismos incrustantes, risco das unidades se desprenderem, instabilidade. Há, ainda, a possibilidade de liberação de resíduos tóxicos, que, apesar de não ser frequente, deve ser monitorada.

- Embarcaçóes desativadas

- Benefícios: Criaçáo de áreas atrativas para a prática de mergulho contemplativo, durabilidade (dependendo do material da embarcação, condição física, local de deposição e condiçôes do mar), alto perfil vertical favorecendo a atração de espécies pelágicas e demersais.

- Prejuízos: Criação de conflito entre mergulhadores e pescadores, alto custo de limpeza e fundeio, risco de acidente por desprendimento de peças, risco de atrapalhar rotas de navegação, muitas vezes há necessidade de sinalização na superfície, dificuldade de implantaçáo no local e posiçáo desejados e dificuldade de limpeza para remoção de quaisquer substâncias tóxicas. Há ainda efeitos nocivos da utilização de explosivos durante o processo de fundeio, que pode enfraquecer a estrutura da embarcação, gerar detritos e colocar em risco a vida marinha.

Collins et al (2002) investigaram a possibilidade de liberaçáo de compostos químicos por estruturas de concreto e pneus na biota incrustante.

Além da composiçáo do material empregado na configuração das estruturas, os recifes artificiais podem ter sua origem dividida em materiais sucateados (ou de oportunidade), como carcaças e agregados de pneus, e materiais especialmente confeccionados para utilização como habitats artificiais, como, por exemplo, os "Reef Balls". O concreto é um dos materiais mais utilizados mundialmente, assim como rochas, módulos de pneus e embarcaçóes ou plataformas desativadas (Baine, 2001).

Materiais sucateados têm como vantagens a pronta disponibilização e o baixo custo de aquisição, mas podem não ser táo resistentes e eficientes quanto as estruturas especialmente desenhadas. Brock e Norris (1989) constataram que materiais sucateados despejados ao acaso fornecem um menor incremento de biomassa em relação a módulos implantados cuidadosamente e especialmente desenhados, além de possuírem baixa estabilidade e expectativa de vida. Quintero (2009) afirma que os módulos recifais construídos com pneus inservíveis em seu trabalho apresentaram resistência e estabilidade satisfatória, o que está diretamente relacionado à quantidade de pneus por módulo.

Emalguns países, comoo Japão, asestruturassãodesenhadas e construídas por engenheiros, com materiais duráveis, préfabricados e colocados em locais cientificamente escolhidos, em águas rasas e profundas, sendo utilizados principalmente para a pesca comercial (Bohnsack e Sutherland, 1985). Já nos projetos norte-americanos predomina a utilização materiais sucateados, de baixo custo, instalados por voluntários interessados no projeto, em áreas mais profundas, longe da costa e são utilizados principalmente por pescadores esportivos embarcados (Bohnsack e Sutherland, 1985).

$\mathrm{O}$ recife deve ser desenhado e confeccionado levando em consideração o material disponível e as necessidades de habitat das espécies que se deseja associar (Nakamura, 1985; ASMFC, 1998). No entanto, Baine (2001) alerta que não é simples fazer uma comparação dos modelos de RAECs possíveis, dada a singularidade de cada ambiente onde este pode ser instalado. Apesar disso, Bortone (2006) afirma que a maioria dos estudos busca criar modelos ecológicos baseados na implantação de um RAEC especialmente desenhado. As estratégias para o planejamento do tamanho e instalaçáo de RAECs estão sujeitas a julgamento científico que consideram a dinâmica oceanográfica, as características de migração dos peixes, o comportamento durante o ciclo de vida e as metodologias de exploração dos recursos (Nakamura, 1985). Athiê (1999) elenca como fatores importantes a serem observados nos materiais de construção de RAECs: a durabilidade, o custo, a resistência à circulação da água, a área de superfície, a facilidade de montagem e embarque e tempo de mão de obra. 
A área superficial total é proporcional à biomassa que o recife pode suportar, uma vez que permite o crescimento de organismos incrustantes, que servem de alimento para diversas espécies de peixes. Aberturas no recife podem ajudar a aumentar esta área, e estes orifícios também são importantes para a circulação de água no interior do recife, evitando a estagnação, que poderia causar uma deficiência no aporte de nutrientes, larvas e oxigênio, criando áreas de anoxia no interior das estruturas (ASMFC, 1998). Outro fator que pode realçar o assentamento de organismos incrustantes é a rugosidade da superfície do recife (Bohnsack et al., 1991; Gratwicke e Speight, 2005).

Segundo Baine (2001), o perfil vertical interfere no tipo de espécies atraídas para as estruturas. Perfis altos favorecem espécies migratórias, de meia água e superfície, enquanto um perfil baixo, com maior extensão horizontal, favorece a espécies demersais. $\mathrm{O}$ autor menciona a atração de moluscos de conchas móveis para estruturas de perfil menor. Bohnsack e Sutherland (1985), revisando a literatura, apontam que estruturas com paredes laterais quase verticais sáo consideradas muito eficientes por produzirem sons atrativos e criarem áreas protegidas da corrente. Zalmon et al. (2002) e Rilov e Benayahu (2002) associam maior riqueza de espécies à complexidade da estrutura e área vertical disponível. Kellison e Sedberry (1998) encontraram maior abundância de peixes demersais em módulos de recifes artificiais com maior perfil vertical, talvez pelas estruturas serem mais visíveis aos peixes ou por tê-los atraído por sons de baixa freqüência emitidos pelos dispositivos de agregação usados.

Sherman et al. (2002) não observaram influência na riqueza de espécies em decorrência do uso ou não de um atrativo flutuante, apesar dos mesmos autores relatarem que essas estruturas podem aumentar o recrutamento. Rilov e Benayahu (2002) verificaram recrutamento nas porçôes mais altas de um recife artificial, mostrando a importância de estruturas verticais para o assentamento de espécies.

Brock e Norris (1989) sugerem uma relação inversa entre a dispersão das estruturas e o tamanho médio dos peixes encontrados. Nakamura (1985) sugere que as estruturas devem estar a menos de mil metros de distância para que os peixes transitem de uma a outra e coloca como sendo duzentos metros a distância ideal para organismos demersobentônicos e trezentos metros para peixes pelágicos.

Uma variável de habitat que afeta o assentamento, a sobrevivência nos estágios iniciais do ciclo de vida e interações pós-recrutamento é o abrigo fornecido pelas estruturas (Hixon e Beets, 1989). Kellison e Sedberry (1998) consideram que a presença de locas tem um efeito positivo no número de espécies, mas o diâmetro das locas presentes nas estruturas foi um fator significante apenas ocasionalmente no número de indivíduos e espécies demersais e que não influenciou significantemente o tamanho médio estimado das espécies. No entanto, Sherman et al. (2002) apontam que gestores devem se atentar ao tamanho das locas disponibilizadas em um RAEC considerando as fases de vida das espécies que farão uso da área.

Hixon e Beets (1989) concordam que a abundância de locas favorece a eficácia do recife, mas encontraram um incremento de peixes piscívoros grandes devido à presença de locas maiores, causando uma queda no número de peixes menores. Isso sugere que estruturas implantadas para serem áreas de pesca constantes devem incluir locas pequenas, para servirem de refúgio contra predação, e locas grandes para as espécies-alvo. Miller (2002) afirma que recifes artificiais com maior disponibilidade e heterogeneidade de refúgios permitem uma maior abundância de peixes.

Kellison e Sedberry (1998) acreditam que esta diferença de resultados se deva a um aumento não suficiente de tamanho nas maiores locas e sugerem que haja, portanto, um tamanho limite no diâmetro das locas para que estas sejam consideradas pequenas e agreguem peixes pequenos. Nakamura (1985) sugere que as aberturas nas estruturas não ultrapassem dois metros de diâmetro sob risco do peixe não perceber sua presença.

\section{B) Hidrografia}

Aspectos da dinâmica oceanográfica local, como regime de correntes, altura e período potencial das ondas e profundidade devem ser levados em consideração na implantação de projetos utilizando RAECs (ASMFC, 1998), uma vez que podem interferir no assentamento, colonização e desempenho geral das estruturas.

Morley et al. (2008) relatam os problemas encontrados na Flórida (EUA) com a implantação de RAECs formados de pneus que não resistiram às tempestades tropicais e aos ocasionais furacôes da região, sendo dispersos até vários quilômetros de sua origem.

A profundidade da coluna d'água deve ser considerada por diversos aspectos. A distribuição de diversas espécies e a quantidade de luz incidente, que afeta as taxas de crescimento e a colonização de numerosos organismos bentônicos, estão relacionadas à profundidade (Bohnsack et al. 1991; Bortone e Kimmel, 1991). Moffit et al. (1989) consideram a profundidade mais importante que o material do recife e sua configuração na determinação de biomassa de espécies transitórias. Para Tseng et al. (2001), usando um sistema de informaçóes geográficas para escolher um local para implantar um RAEC, o parâmetro de profundidade da coluna d'água foi o mais significativo para obter resultados positivos. Apesar de recifes rasos serem mais propícios a uma melhor produtividade primária, deve-se considerar o espaço entre o topo do recife e a superfície da água para não atrapalhar rotas de navegação (Baynes e Szmant, 1989).

Turbidez, salinidade e níveis de poluição podem afetar o perfil de organismos nas estruturas, dependendo da tolerância das espécies a estes fatores. Peixes atraídos para RAECs por orientação visual podem ser afetados por uma elevada turbidez da água (Bohnsack et al. 1991). Temperatura, quantidade de nutrientes, oxigênio, carbono particulado, pesticidas e metais pesados também são relevantes (Bortone e Kimmel, 1991).

A exposição às correntes aumenta a exposição do recife a recrutas larvais e pode aumentar a oferta de alimento (Baynes e Szmant, 1989; Bortone e Kimmel, 1991; ASMFC, 1998; Sherman et al. 1999). Em geral, áreas com fluxos mais velozes e com baixa sedimentação correspondem a regióes de maior diversidade de espécies (Baynes e Szmant, op cit). Deve-se considerar que a proximidade de fontes de recrutas em distância real pode náo significar uma proximidade maior na prática, uma vez que correntes podem transportar 
para áreas distantes com maior eficiência do que para áreas vizinhas (Bortone e Kimmel, 1991).

Baynes e Szmant (1989) recomendam que, para que se obtenha um maior crescimento da comunidade bentônica, as estruturas artificiais sejam instaladas de modo a maximizar a quantidade de área superficial exposta ao fluxo laminar da corrente e a quantidade de substrato vertical. Os autores alertam para o fato de correntes com velocidades muito elevadas causarem a retração de tentáculos utilizados na alimentação, a remoção do substrato e a diminuição do assentamento larval. Uma movimentação excessiva de água pode afetar a configuração das estruturas, causando deslizamento, soterramento ou quebra de partes estruturais (Grove et al., 1991; ASMFC, 1998).

No caso de RAECs desenvolvidos para a proteção da linha de costa e melhora no surfe, Voorde et al. (2008) listam como aspectos importantes a serem considerados na geometria da estrutura: altura de onda existente e projetada, profundidade de arrebentação, nível da maré e distância da linha de costa.

\section{C) Granulometria}

Análises da composição de tamanho do sedimento de fundo na área em que o recife será instalado são fundamentais para a implantaçâo do projeto. Alguns tipos de sedimentos podem não ser compatíveis com o material e modelo utilizado na construção do recife, soterrando as estruturas ou dificultando seu assentamento, permitindo que se movam excessivamente (NOAA, 1985).

A movimentação do sedimento é inevitável quando a velocidade das partículas de água excede a velocidade limite de um determinado tamanho de partícula de sedimento. Em áreas sujeitas a uma grande mobilidade do sedimento é recomendável que os blocos descansem sobre três ou quatro pontos de contato com o substrato. Contato plano entre os módulos e o substrato pode levar à escavaçáo lateral da área e eventual tombamento da estrutura (Grove et al., 1991). Em casos de fundos compostos por sedimentos muito macios (lamosos), a estrutura pode afundar no substrato por compactar a lama devido a seu peso. Nestes casos o contato plano é desejável (JCFPA, 1986 apud Grove et al., op. cit.).

$\mathrm{O}$ declive do fundo também é relevante, fundos com declives pouco íngremes são recomendados. Outra consideração importante a respeito do tipo de sedimento do fundo se refere às partículas que se encontram em suspensão e que podem ser depositados nas estruturas do recife, prejudicando organismos incrustantes filtradores (Matthews, 1985; NOAA, 1995). As propriedades do substrato influenciarão o tipo e a abundância de organismos bentônicos que servirão como alimento (Bortone e Kimmel, 1991).

\section{D) Teoria da Agregação versus Produção de Nova Biomassa}

Uma das maiores polêmicas em relação ao uso de RAECs, especialmente com fins de incremento da atividade pesqueira, diz respeito ao efeito da instalação destes empreendimentos no desenvolvimento da comunidade biológica. Ainda não é clara qual a função predominante dos RAECs: a produçáo de nova biomassa ou a simples agregação dos recursos (Bohnsack, 1989; Polovina, 1991; Powers et al., 2003). Apesar de estas hipóteses muitas vezes serem consideradas mutuamente exclusivas, elas podem ser interpretadas também como dois extremos de um gradiente (Svane e Petersen, 2002; Osenberg et al., 2002).

Em estruturas artificiais submetidas à pressão pesqueira esta questáo torna-se extremamente relevante, uma vez que a captura acentuada de pescado promovida pelos RAECs pode conduzir o estoque explorado à sobrepesca quando as estruturas funcionam simplesmente redirecionando o estoque e não há controle da utilização da área pelos pescadores (Bohnsack, 1989; Polovina, 1991).

Segundo Bohnsack (1989), os fatores considerados importantes na questáo agregação versus produção de nova biomassa são a disponibilidade de recifes naturais, os mecanismos naturais de limitação populacional, a pressão da exploração pesqueira, a dependência de recifes durante o ciclo de vida e as características comportamentais da espécie e a idade do organismo. Ainda segundo o autor, o aumento da produtividade é mais provável em locais isolados de recifes naturais e para espécies obrigatoriamente recifais, limitadas por habitat, demersais, filopátricas e territorialistas. Enquanto a atração deve prevalecer em locais com abundância de habitats recifais naturais, onde taxas de exploração são altas e para espécies pelágicas, limitadas por recrutamento, com alta mobilidade, parcialmente dependentes dos recifes e oportunistas. Osenberg et al. (2002) também consideram que o incremento de biomassa de pequenos peixes deve ser provindo de um redirecionamento larval e náo de sua migração.

Segundo White et al. (1990), os recifes demersais em águas tropicais rasas fariam aumentar, pelo menos, a produção primária de algas e invertebrados, possivelmente aumentando o habitat natural para suportar maior biomassa de peixes. Hixon e Beets (1989) colocam que o abrigo pode ser limitante para uma fase do ciclo de vida e não para a outra, assim estruturas artificiais agiriam de forma diferente dependendo da etapa do ciclo de vida da espécie.

Polovina (1991) define três formas de atuação de RAECs sobre os recursos pesqueiros. Na primeira há a redistribuição da biomassa explorada, sem aumentá-la ou o tamanho total do estoque. Na segunda ocorre agregação de biomassa anteriormente náo explorada, aumentando sua exploração, e na terceira há o real aumento da biomassa total. Como exemplo dos dois primeiros casos, o autor cita os peixes de comportamento transitório, que não permanecem nos recifes por tempo prolongado e as estruturas artificiais agem redirecionando a biomassa, não havendo alteraçóes significativas na captura quando a área de pesca total é considerada. O impacto de RAECs, nestes casos, é uma redução na biomassa explorável se não houver controle da pressão pesqueira sobre a estrutura artificial. Ainda segundo o autor, o aumento da densidade pode gerar o aumento da capturabilidade do petrecho e a facilidade do acesso pode aumentar o esforço, podendo aumentar a mortalidade. Quanto ao terceiro caso, segundo o autor, o fornecimento de habitat adicional poderia aumentar o tamanho populacional de alguns estoques limitados pela disponibilidade de habitat, por melhorar o assentamento larval, o crescimento juvenil e reduzir a predaçấo. Deve-se considerar, neste caso, o quanto a 
nova produção de biomassa gerada pelas estruturas artificiais pode suportar o aumento da pressão pesqueira na área.

Polovina (1991) e Bohnsack (1989) sugerem que há pouca evidência que RAECs aumentem substancialmente o estoque de organismos marinhos. Hixon e Beets (1989) dizem que a limitação pela disponibilidade de abrigo parece não funcionar em locais onde a disponibilidade de recrutas larvais é pequena. Moffit et al. (1989) sugerem que recifes artificiais de pequena escala profundos funcionam primariamente como instrumentos de agregação de pescado e nâo aumentando a produçấo, devendo ser utilizados para agregar espécies-alvo cujo estoque se encontra subexplotado. Segundo esses autores, estes recifes quando utilizados na agregação de estoques ameaçados com uma atividade pesqueira não controlada podem ampliar ainda mais o problema, ao invés de fornecer soluçóes.

Bohnsack (1989) diz que estruturas artificiais tendem a não beneficiar populaçóes fortemente exploradas ou em sobrepesca. Uma consideração interessante é que, no caso de estoques já em sobrepesca, a disponibilidade de habitat não deve mais ser o fator limitante e sim a própria pressão pesqueira. D’Anna et al. (1994) relata que a escassez de macroalgas fez com que o recife artificial funcionasse apenas para atrair e concentrar espécies abundantes e freqüentes, receber temporariamente grandes predadores que encontram refúgio durante a alimentação nas estruturas e atrair espécies pelágicas. Kellison e Sedberry (1998) também consideraram que os peixes foram atraídos para estruturas artificiais.

A maior parte dos indivíduos recrutados para os habitats artificiais são adultos ou subadultos, sugerindo agregação (Matthews, 1985; Moffit et al., 1989). A presença de sazonalidade na composiçâo das espécies associadas a RAECs notada por diversos autores pode contribuir para a hipótese de agregação de biomassa, uma vez que estas espécies não seriam residentes nas estruturas artificiais. No entanto, Hixon e Beets (1989) realizaram estudos de marcaçâo dos peixes nas estruturas artificiais e os indivíduos marcados foram constantemente observados no local, sugerindo residência. Matthews (1985) encontrou uma grande movimentação de peixes dos recifes naturais para os artificiais, mas não o contrário, sugerindo agregação, mesmo para espécies tidas como sedentárias.

Outra consideração relevante é o fato das espécies transitórias possuírem uma maior importância econômica em preço por quilo e biomassa atraída para os recifes (Moffit et al., 1989), o que as coloca como alvo da atividade pesqueira. Segundo D’Anna et al. (1994) o maior número de indivíduos em estruturas artificiais foi devido à abundância maior de espécies que, geralmente, possuem tamanho pequeno. Assim, mesmo que os habitats artificiais estejam produzindo biomassa de peixes recifais, estes podem não ser o alvo da atividade pesqueira que se queria incrementar. No entanto, novos recursos explorados nos RAECs podem obter um maior valor de mercado no comércio local, aumentado o lucro da atividade (Milon, 1989).

Um melhor entendimento da importância relativa da função de produtores ou agregadores das estruturas artificiais é crucial para um gerenciamento pesqueiro coerente $\mathrm{e}$ para construção de RAECs eficazes (Bohnsack, 1989). Para resolver este debate são necessárias abordagens mais integradoras que reconheçam os efeitos de vários processos e que utilizem técnicas diferentes, procurando investigar a influência que um recife natural pode ter em um sistema artificial (Osenberg et al., 2002).

\section{E) Comunidade biológica}

A implantação de estruturas artificiais em fundos arenosos desertos representa um elemento de descontinuidade na biocenose do substrato macio (D'Anna et al., 1994), uma vez que, seja por agregação ou produção de biomassa, concentram grande quantidade de organismos. Essa alteração na composição de espécies da área de implantaçáo dos RAECs deve ser analisada para que possíveis impactos negativos possam ser detectados e contornados. Polovina (1991) afirma que RAECs alteram a composição e abundância de espécies em áreas pesqueiras e modificação das espécies alvo. Algumas áreas desertas podem ter uma função ecológica, agindo como barreiras para a distribuição de certas espécies ou ainda de populaçóes diferentes de determinada espécie. Apesar de que um único RAEC não seja significativo em tamanho, quando comparado à plataforma continental como um todo, o acúmulo de instalaçôes pode acarretar mudanças ecológicas importantes (Bortone, 2006).

Um fator a ser considerado é a possibilidade das estruturas inseridas artificialmente no fundo marinho funcionarem como substrato de fixação e posterior alastramento de espécies exóticas ou invasoras. Ferreira et al. (2004) identificam plataformas de petróleo como vetores de contaminação de espécies exóticas oriundas de água de lastro. A instalação de RAECs em áreas de pesca, distantes da costa e com grande fluxo de embarcaçóes poderia agir da mesma forma. Inclusive, permitindo que espécies invasoras, que normalmente não conseguiriam atingir uma área de recife natural devido ao seu percurso e tempo de estágio larval, possam, com o novo substrato servindo como ponte, conseguir disseminar-se para ambientes recifais naturais de áreas adjacentes.

A movimentação de peixes dos recifes naturais para os artificiais, mas não o inverso, observado por Matthews (1985) e corroborado por D’Anna et al. (1994), sugere que a colonização inicial das estruturas por adultos e subadultos, aliado à grande pressão pesqueira nos recifes artificiais pode ter efeitos negativos nas populaçóes de peixes dos recifes naturais adjacentes. Assim, recomenda-se que RAECs sejam implantados longe de recifes naturais. Bohnsack (1989) concorda com esta afirmação, porém Stone et al. (1989 apud D’Anna et al., 1994) não notou influências negativas.

\section{F) Utilização das estruturas}

O mar é uma área de domínio público e uso comum (Pickering, 1996) e, portanto, a implantação de estruturas que possam alterar de algum modo a utilização deste recurso pela sociedade, como RAECs, é uma questão que merece atenção. A elaboração e a instalação deste tipo de empreendimento devem ser debatidas com a participação de membros da comunidade local de diversos grupos (Conceição e Franklin-Júnior, 2001). Os autores sugerem que sejam realizadas atividades de capacitação sobre os fundamentos técnicos, ecológicos, sociais e econômicos das estruturas. Os objetivos do projeto devem ser compatíveis com as atividades previamente desenvolvidas no local. 
As diferentes funçóes que os RAECs podem exercer também podem ser incompatíveis entre si, gerando conflito entre grupos de usuários distintos caso não haja uma definição clara dos objetivos de implantação do projeto (Polovina, 1991; Santos et al., 2010). Os usuários do local devem estar cientes destes objetivos e concordar com a implantação. Dentro do mesmo grupo de usuários ou outro grupo compatível, a implantação de RAECs pode gerar conflitos decorrentes da competição pela utilização da área. Em casos de RAECs voltados à atividade pesqueira, o uso das estruturas em demasia pode gerar uma série de prejuízos decorrentes da competiçáo pelo pescado.

Um caso interessante é o do estado de Pernambuco, onde há um grande número de naufrágios que hoje são classificados como RAECs. Santos et al. (2010) explicam que, inicialmente, pescadores e mergulhadores usavam a área, porém com uma mudança na legislação a pesca se tornou proibida, causando grande descontentamento na frota artesanal. Como resposta, estes usuários continuam suas atividades na regiáo agora de maneira ilegal. Os autores afirmam que esta legislação deveria estar voltada para um desenvolvimento sustentável e equitativo.

RAECs podem servir para redistribuir o esforço de áreas congestionadas, mitigando a competição pelos recursos (Polovina, 1991). Por outro lado, o congestionamento no local de implantaçáo das estruturas pode gerar conflitos de petrechos e problemas com os demais equipamentos (Milon, 1989). Ainda de acordo com o autor, o desempenho econômico geral na atividade pesqueira pode ser enfraquecido caso a atração exercida pelas estruturas venha a causar sobrepesca ou o conflito de usuários aumente os custos de captura. Um aumento descontrolado do esforço de pesca na área de atuação dos RAECs pode levar à sobrepesca dos recursos existentes, especialmente se as estruturas funcionarem primariamente como agregadores de pescado (Milon, 1989; Polovina, 1991).

Além disso, projetos com cada um dos diferentes objetivos de instalação devem possuir algumas características que facilitem sua utilização e ajudem a selecionar seu grupo de usuários. Estruturas direcionadas à prática de mergulho contemplativo, por exemplo, devem estar acomodadas no fundo de modo estável, preferencialmente sem pontas ou superfícies cortantes ou com risco de desprendimento, para evitar acidentes durante o mergulho. Estes também devem estar situados em profundidades até 40 metros (de acordo com os limites impostos pelas certificadoras internacionais de mergulho autônomo), em águas com boa visibilidade e correntes suaves durante a maior parte do ano e distante de áreas tradicionalmente de pesca, para evitar conflito de usuários. Projetos instalados para pesquisa científica a respeito do funcionamento das estruturas devem ser instalados em áreas com uso restrito, para que não haja interferência da ação não controlada da população. No caso de RAECs voltados à atividade pesqueira, as estruturas devem ser colocadas em áreas próximas a locais de desembarque e com distância da costa adequada ao tipo de embarcação que irá utilizá-las (Polovina, 1991).

No Brasil, ainda não há legislação vigente sobre a instalação de RAECs. O Projeto de Lei No 3.929, de 2004, preenche esta lacuna, especificando as finalidades de um recife artificial, condiçóes de licenciamento às quais estão sujeitas uma instalação, inclusive em unidades de conservação, penalidade para os infratores (referente à Lei de Crimes Ambientais) e a cláusula de vigência.

Bortone (2006) afirma que estudos com RAECs ainda apresentam progresso lento. Porém, avanços como o uso de pacotes estatísticos não-paramétricos e multivariados, melhor delineamento dos estudos, permitindo maior reprodutibilidade e acurácia, têm sido tendências que deverão aparecer nos próximos anos.

\section{CONCLUSÓES}

Recifes Artificiais e Estruturas Correlatas podem ser vistos como ferramentas interessantes no manejo de áreas costeiras marinhas. No entanto, a implantaçáo deste tipo de empreendimento deve ser planejada com extrema cautela, levando em consideração diversos fatores que podem pôr em risco a eficácia das estruturas ou, ainda, o meio em que foram instaladas.

Aspectos da dinâmica oceanográfica, da composição do sedimento, da configuração das estruturas e das comunidades biológicas locais e adjacentes devem ser avaliados para um bom funcionamento do sistema de RAECs.

Os objetivos da implantação devem ser claros e condizentes com as atividades costumeiras da região para que não haja conflito entre diferentes grupos de usuários.

No caso de estruturas voltadas para atividades pesqueiras, há necessidade de ainda maior cautela e monitoramento após a instalação, devido à possibilidade de impacto nos estoques pesqueiros. Assim, estes empreendimentos devem estar dentro de um plano de gerenciamento mais amplo, com sua utilização regulamentada e acompanhamento da situação dos estoques e da produção pesqueira após a instalação, previsto.

Portanto, antes de iniciar um projeto de implantação de recifes artificiais é importante considerar a relevância da utilização destas estruturas, seus riscos e compensaçóes, realizando um planejamento cuidadoso de cada etapa.

\section{BILIOGRAFIA}

ASMFC (1998) - Coastal Artificial Reef Planning Guide. 545p., The Joint Artificial Reef Technical Committee of the Atlantic and Gulf States Marine Fisheries Commissions (ASMFC), Ocean Springs, MS, U.S.A. http://www.gsmfc.org/publications/Miscellaneous/ Coastal_Artificial_Reef_Planning_Guide_1998.pdf

Athiê, A.A.R. (1999) - Colonização e sucessão ecológica de peixes de recifes artificiais no Canal de São Sebastiāo, Litoral Norte do Estado de São Paulo- Brasil. 192p., Dissertação de Mestrado, Universidade de São Paulo, São Paulo, SP, Brasil. Não publicado.

Baine, M. (2001) - Artificial reefs: a review of their design, application, management and performance. Ocean \& Coastal Management, 44(3-4):241-259. DOI 10.1016/ S0964-5691(01)00048-5.

Barber, J.S.; Chosid, D.M.; Glenn, R.P.; Whitmore, K.A. (2009) - A systematic model for artificial reef site selection. New Zealand Journal of Marine \& Freshwater Research, 43:283-297. DOI: 0028-8330/09/4301-0283. 
Baynes, T.W.; Szmant, A.M. (1989) - Effect of current on the sessile benthic community structure of an artificial reef. Bulletin of Marine Science (ISSN: 0007-4977), 44(2):545-566, Miami, FL, U.S.A. Disponível em: http://www.ingentaconnect.com/content/umrsmas/ bullmar/1989/00000044/00000002/art00003.

Bohnsack, J.A.; Johnson, D.L.; Ambrose, R.F. (1991) - Ecology of Artificial Reef Habitats and Fishes. In: Seaman, W. Jr. \& Sprague, L.M. Artificial Habitats for Marine and Freshwater Fisheries, pp.61-107, Academic Press, San Diego, EUA. ISBN: 0126343454.

Bohnsack, J.A.; Sutherland, D.L. (1985) - Artificial reef research: a review with recommendations for future priorities. Bulletin of Marine Science (ISSN: 00074977), 37(1):11-39, Miami, FL, U.S.A.. Disponível em: http://www.ingentaconnect.com/content/umrsmas/ bullmar/1985/00000037/00000001/art00003.

Bonhsack, J.A. (1989) - Are high densities of fishes at artificial reefs the result of habitat limitation or behavioral preference? Bulletin of Marine Science (ISSN: 00074977), 44(2):631-645, Miami, FL, U.S.A.. Disponível em: http://www.ingentaconnect.com/content/umrsmas/ bullmar/1989/00000044/00000002/art00009.

Bortone, S.A. (2006) - A Perspective of artificial reef research: the past, present and future. Bulletin of Marine Science (ISSN: 0007-4977), 78(1):1-8, Miami, FL, U.S.A.. Disponível em: http://www.ingentaconnect.com/ content/umrsmas/bullmar/2006/00000078/00000001/ $\operatorname{art} 00001$.

Bortone, S.A.; Kimmel, J.J. (1991) - Environmental assessment and monitoring of artificial habitats. In: Seaman, W. Jr.; Sprague, L.M., Artificial Habitats for Marine and Freshwater Fisheries, pp.177-236, Academic Press, San Diego, EUA. ISBN: 0126343454.

Brock, R.E.; Norris, J.E. (1989) - An analysis of the efficacy of four artificial reef designs in tropical waters. Bulletin of Marine Science (ISSN: 0007-4977), 44(2):934-941, Miami, FL, U.S.A. Disponível em: http://www.ingentaconnect.com/content/umrsmas/ bullmar/1989/00000044/00000002/art00037.

Claudet, J.; Pelletier, D. (2004) - Marine protected areas and artificial reefs: A review of the interactions between management and scientific studies. Aquatic Living Resources, 17:129-138. DOI: 10.1051/alr:2004017.

Collins, K.J.; Jensen, A.C.; Mallinson, J.J.; Roenelle, V.; Smith, I.P. (2002) - Environmental impact assessment of a scrap tyre artificial reef. ICES Journal of Marine Science, 59: S243-S249. DOI: 10.1006/jmsc.2002.1297.

Conceição, R.N.L.; Franklin-Júnior, W. (2001) - A situação atual dos recifes artificiais instalados na plataforma continental do estado do Ceará, Brasil. Arquivos de Ciências do Mar (ISSN: 0374-5686), 34:107-115, Fortaleza, CE, Brasil. Disponível em: http://www. labomar.ufc.br/images/stories/arquivos/ArqCienMar/ V34_2001/acm_2001_34_13.pdf.

Conceição, R.N.L.(2003) - Ecologia de peixes em recifes artificiais de pneus instalados na costa do Estado do Ceará. $99 \mathrm{p}$. , Tese de doutorado, Universidade Federal de São Carlos, SP, Brasil. Disponível em: http://www.adital.
com.br/banners/Texto $\% 20 \mathrm{~b} \% \mathrm{E} 1$ sico $\% 20$ sobre $\% 20$ Recifes\%20Artificiais.pdf

D’Anna, G.; Badalamenti, F.; Gristina, M.; Pipitone, C. (1994) - Influence of artificial reefs on coastal nekton assemblages of the Gulf of Castellammare (Northwest Sicily). Bulletin of Marine Science (ISSN: 0007-4977), 55(2-3):418-433, Miami, FL, U.S.A. Disponível em: http://www.ingentaconnect.com/content/umrsmas/ bullmar/1994/00000055/F0020002/art00015.

Ferreira, C.E.L.; Gonçalves, J.E.A.; Coutinho, R. (2004) Cascos de navios e plataformas como vetores na introdução de espécies exóticas. In: Silva, J.S.V. \& Souza, R.C.C.L., Água de Lastro e Bioinvasão, pp.143-155, Interciência, Rio de Janeiro, Brasil. ISBN: 8571931003.

Gratwicke, B.; Speight, M.R. (2005) - Effects of habitat complexity on Caribbean marine fish assemblages. Marine Ecology Progress Series, 292:301-310. DOI: $10.3354 / \mathrm{meps} 292301$.

Grove, S.R.; Sonu, C.J.; Nakamura, M. (1991) - Design and engineering of manufactured habitats for fisheries enhancement. In: Seaman, W. Jr. \& Sprague, L.M., Artificial Habitats for Marine and Freshwater Fisheries, pp.109-152, Academic Press, San Diego, EUA. ISBN: 0126343454.

Hixon, M.A.; Beets, J.P. (1989) - Shelter characteristics and Caribbean fish assemblages: experiments with artificial reefs. Bulletin of Marine Science (ISSN: 00074977), 44(2):666-680, Miami, FL, U.S.A. Disponível em: http://www.ingentaconnect.com/content/umrsmas/ bullmar/1989/00000044/00000002/art00012.

Kellison, G.T.; Sedberry, G.R. (1998) - The effects of artificial reef vertical profile and hole diameter on fishes off South Carolina. Bulletin of Marine Science (ISSN: 00074977), 62(3):763-780, Miami, FL, U.S.A. Disponível em http://www.ingentaconnect.com/content/umrsmas/ bullmar/1998/00000062/00000003/art00005.

Lukens, R.R. (coord.) (1997) - Guidelines for marine artificial reef materials. Gulf States Marine Fisheries Commission, 118p. Disponível em http://bay.ifas.ufl. edu/pdfs/sea_grant/Guidelines_for_Marine_Artificial_ Reef_Materials_January_1997.pdf

Matthews, K.R. (1985) - Species similarity and movement of fishes on natural and artificial reefs in Monterey Bay, California. Bulletin of Marine Science (ISSN: 00074977), 37(1):252-270, Miami, FL, U.S.A. Disponível em: http://www.ingentaconnect.com/content/umrsmas/ bullmar/1985/00000037/00000001/art00019.

Miller, M.W. (2002) - Using ecological processes to advance artificial reef goals. ICES Journal of Marine Science, 59:S27-S31. DOI: 10.1006/jmsc.2001.1162.

Milon, J.W. (1989) - Economic evaluation of artificial habitat for fisheries: Progress and challenges. Bulletin of Marine Science (ISSN: 0007-4977), 44(2):831-843. Disponível em: http://www.ingentaconnect.com/content/umrsmas/ bullmar/1989/00000044/00000002/art00027.

Moffit, R.B.; Parrish, F.A.; Polovina, J.J. (1989) - Community structure, biomass and productivity of deepwater artificial reefs in Hawaii. Bulletin of Marine Science (ISSN: 00074977), 44(2):616-630, Miami, FL, U.S.A.. Disponível 
em: http://www.ingentaconnect.com/content/umrsmas/ bullmar/1989/00000044/00000002/art00008.

Morley, D.M.; Sherman, R.L.; Jordan, L.K.B.; Banks, K.W.; Quinn, T.P.; Spieler, R.E. (2008) - Environmental enhancement gone awry: characterization of an artificial reef constructed from waste vehicle tires. WIT Transactions on The Built Environment, 99:73-87. DOI: 10.2495/ CENV080071.

Nakamura, M. (1985) - Evolution of artificial fishing reef concepts in Japan. Bulletin of Marine Science (ISSN: $0007-$ 4977), 37(1):271-278, Miami, FL, U.S.A. Disponível em: http:/www.ingentaconnect.com/content/umrsmas/ bullmar/1985/00000037/00000001/art00020.

NOAA. (1985) - National artificial reef plan. 39p., NOAA - National Oceanic and Atmospheric Adminstration, Technical Memorandum NMFS OF- 06, Washington, DC, U.S.A. Não publicado.

Osenberg, C.W.; Saint Mary, C.M.; Wilson, J.A.; Lindberg, W.J. (2002) - A quantitative framework to evaluate the attraction-production controversy. ICES Journal of Marine Science, 59:S214-221. DOI: 10.1006/ jmsc.2002.1222.

Pickering, H. (1996) - Legal framework governing artificial reefs in the EU. 36p., Portsmouth University, Centre for the Economics and Management of Aquatic Resources (CEMARE), Portsmouth, United Kingdom. Não publicado.

Pitcher, T.J.; Seaman, W. Jr. (2001) - Petrarch's Principle: how protected human-made reefs can help the reconstruction of fisheries and marine ecosystems. Fish and Fisheries, 1(1):73-81. DOI: 10.1046/j.1467-2979.2000.00010.x.

Polovina, J.J. (1991) - Fisheries applications and biological impacts of artificial habitats. In: Seaman, W. Jr. \& Sprague, L.M., Artificial Habitats for Marine and Freshwater Fisheries, pp.153-176, Academic Press, San Diego, EUA. ISBN: 0126343454.

Powers, S.P.; Grabowski, H.; Peterson, C.H.; Lindberg, W.J. (2003) - Estimating enhancement of fish production by offshore artificial reefs: uncertainty exhibited by divergent scenarios. Marine Ecology Progress Series, 264:265-277. DOI: $10.3354 /$ meps264265.

Quintero, E.C.H. (2009) - Construçâo e implantação de recifes artificiais em comunidades pesqueiras, usando pneus inservíveis. 113p., Dissertação de Mestrado, Universidade Federal da Bahia, Salvador, BA, Brasil. Não publicado.

Rilov, G.; Benayahu, Y. (2002) - Rehabilitation of Coral Reef-Fish Communities: The Importance of ArtificialReef Relief to Recruitment Rates. Bulletin of Marine Science (ISSN: 0007-4977), 70(1):185-197, Miami, FL, U.S.A. Disponível em: http://www.ingentaconnect.com/ content/umrsmas/bullmar/2002/00000070/00000001/ $\operatorname{art} 00015$.

Santos, D.H.C.; Cunha, M.G.G.S.; Amâncio, F.C.; Passavante, J.Z.O. (2010) - Recifes Artificiais, Mergulho e Pesca Artesanal: Alguns Aspectos do Conflito na Costa de Pernambuco - Brasil. Revista de Gestão Costeira Integrada, 10(1):7-22. http://www.aprh.pt/rgci/pdf/rgci154_Santos.pdf.

Seaman, W.Jr.; Sprague, L.M. (1991a) - Artificial Habitats for Marine and Freshwater Fisheries. 258p., Academic Press, San Diego, EUA. ISBN: 0126343454.
Seaman, W.Jr.; Sprague, L.M. (1991b) - Artificial habitat practices in aquatic systems. In: Seaman, W. Jr. \& Sprague, L.M., Artificial Habitats for Marine and Freshwater Fisheries, pp.1-29, Academic Press, San Diego, EUA. ISBN: 0126343454.

Sherman, R.L.; Gilliam, D.S.; Spieler, R.E. (1999) - A preliminary examination of depth associated spatial variation in fish assemblages on small artificial reefs. Journal of Applied Ichthyology, 15:116-121. DOI: 10.1046/j.1439-0426.1999.00120.x.

Sherman, R.L.; Gilliam, D.S.; Spieler, R.E. (2002) - Artificial reef design: void space, complexity, and attractants. ICES Journal of Marine Science, 59:S196-S200. DOI: 10.1006/ jmsc.2001.1163.

Steimle, F.W.; Méier, M.H. (1997) - What information do artificial reef managers really want from fishery science? Fisheries, 22(4):6-8. DOi: DOI:10.1577/1548-844622-4

Sutton, S.G.; Bushnella, S.L. (2007) - Socio-economic aspects of artificial reefs: Considerations for the Great Barrier Reef Marine Park. Ocean \& Coastal Management, 50(10):829846. DOI: 10.1016/j.ocecoaman.2007.01.003.

Svane, I.; Petersen, J.K. (2002) - On the problem of Epibioses, Fouling and Artificial Reefs, a Review. Marine Ecology, 22(3):169-188. DOI: 10.1046/j.14390485.2001.01729.x.

Tseng, C.T.; Chen, S.C.; Huang, C.S.; Liu, C.C. (2001) - GIS-assisted site selection for artificial reefs. Fisheries Science, 67:1015-1022. DOI: 10.1046/j.14442906.2001.00356.x.

Voorde, M. tem; Neves, M.G.; Antunes do Carmo, J.S. (2008) - Estudo Preliminar da Geometria de um Recife Artificial para Protecção Costeira e para a Prática de Surf na Costa Oeste Portuguesa. Revista de Gestão Costeira Integrada, 8(1):65-79. http://www.aprh.pt/rgci/pdf/ revista8f1_4.pdf.

Voorde, M. ten; Antunes do Carmo, J.S.; Neves, M.G. (2009) - Designing a Preliminary Multifunctional Artificial Reef to Protect the Portuguese Coast. Journal of Coastal Research, 25(1):69-79. DOI: 10.2112/07-0827.1.

White, A.T.; Ming, C.T.; De Silva, M.W.R.N.; Guarin, F.Y. (1990) - Artificial reefs for marine habitat enhancement in Southeast Asia. 45p. Association of Southeast Asian Nations / United States Coastal Resources Management Project. ISBN: 9711022834.

Whitmarsh, D.; Santos, M.N.; Ramos, J.; Monteiro, C.C. (2008) - Marine habitat modification through artificial reefs off the Algarve (southern Portugal): An economic analysis of the fisheries and the prospects for management. Ocean \& Coastal Management, 51:463468. DOI: 10.1016/j.ocecoaman.2008.04.004.

Wilson, K.D.P.; Leung, A.W.Y.; Kennish, R. (2002) Restoration of Hong Kong fisheries through deployment of artificial reefs in marine protected areas. ICES Journal of Marine Science, 59:S157-S163. DOI: 10.1006/ jmsc.2002.1186.

Zalmon, I.R.; Novelli, R.; Gomes, M.P.; Faria, V.V. (2002) - Experimental results of an artificial reef programme on the Brazilian coast north of Rio de Janeiro. ICES Journal of Marine Science, 59:S83-S87. DOI: 10.1006/ jmsc.2002.1273. 